\title{
Estimação e Equalização em Frequência de Sistemas OFDM com Amplificadores de Potência sem Memória
}

\author{
Paulo A. C. AGUILAR, Carlos A. R. FERNANDES, João C. M. MOTA e Gérard FAVIER
}

\begin{abstract}
Resumo- Neste artigo são desenvolvidas técnicas para estimação e equalização de sistemas de comunicações sem fio OFDM com amplificadores de potência sem memória. As nãolinearidades dos amplificadores são responsáveis por interferências entre as sub-portadoras. A proposta para recuperar símbolos transmitidos por bloco no receptor usa inicialmente a estimação LS (Least Squares) do canal global (amplificador + canal sem fio). O canal estimado é usado na equalização MMSE (Minimum Mean Square Error) e ZF (Zero Forcing), considerando a retransmissão dos símbolos com potências diferentes. Os resultados obtidos por simulação indicam o bom desempenho das técnicas propostas.
\end{abstract}

Palavras-Chave- OFDM, estimação de canal não-linear, equalização em frequiência, amplificador de potência, diversidade de potência.

Abstract- This paper develops techniques for estimation and equalization of wireless OFDM communication systems with memoryless power amplifiers, whose non-linearity are responsable for intercarrier interference. The proposed strategy for recovering of the transmitted symbol blocks uses LS (Least Squares) estimation of the global channel (amplifier + wireless channel). The estimated channel is used in the receiver by means of equalization techniques with the MMSE (Minimum Mean Square Error) and ZF (Zero Forcing) criteria, considering the retransmission of the symbols with different powers. The good performance of the proposal is indicated by simulation results.

Keywords- OFDM, nonlinear channel estimation, frequency equalization, power amplifier, power diversity.

\section{INTRODUÇÃO}

Os sistemas de transmissão multi-portadoras, como o sistema OFDM (Orthogonal Frequency Division Multiplexing), em geral mostram uma grande sensibilidade aos efeitos da distorção não-linear causados por amplificadores de potência (PA - Power Amplifier) [1-4]. De fato, um dos maiores problemas associados aos sistemas OFDM é o fato de os sinais transmitidos possuírem uma grande variação de amplitude, ou seja, uma alta PAPR (Peak-to-Average Power Ratio) [1,2,5], fazendo com que algumas componentes do sinal OFDM estejam próximos à zona de saturação do $\mathrm{PA}$, resultando na introdução de interferência entre portadoras (ICI - Intercarrier

Paulo A. C. Aguilar, Carlos A. R. Fernandes e G. Favier, Laboratório I3S/UNSA/CNRS, Sophia Antipolis, França, E-mails: \{paulo, acarlos, favier\} @i3s.unice.fr. João C. M. Mota e Carlos A. R. Fernandes, GTEL/DETI/UFC, Fortaleza, Brasil, E-mails: \{mota, carlosalexandre\}@gtel.ufc.br. Carlos A. R. Fernandes é bolsista da CAPES.
Interference) devido à não-linearidade presente. Já a interferência entre diferentes blocos de transmissão é evitada com a utilização de prefixo cíclico [7].

Este artigo é dedicado ao desenvolvimento e aplicação de técnicas de estimação e equalização de canais OFDM com PA não-linear sem memória, cujo modelo adotado é baseado em polinômio cujos coeficientes complexos introduzem distorções de amplitude e fase ao sinal recebido [8].

Os efeitos das distorções não-lineares em sistemas OFDM foram explorados em diversos trabalhos que propõem diferentes análises [2, 4] e soluções para o problema, como a utilização de técnicas de pré-distorção [8-10] e equalização de canal não-linear $[8,11,12,13]$. É importante ressaltar que este trabalho não utiliza métodos de controle de variação de amplitude, como técnicas de controle de PAPR [14], para atenuar a interferência não-linear do PA. Os métodos de estimação e equalização são baseados numa representação de canal global, que compreende uma cascata entre o canal linear sem fio e o PA. A estimação de canal global proposta é baseada no método LS e utiliza símbolos pilotos conhecidos durante o período de treinamento. Em seguida, assumindo-se que os coeficientes do canal global são conhecidos, propõem-se duas técnicas de equalização em freqüência de canal com critérios MMSE e ZF, as quais são baseadas em um receptor de diversidade em potência (PDR - Power Diversity-based Receiver), o qual prevê a recepção de símbolos transmitidos com potência diferentes. Será demonstrado que, devido à natureza não-linear do canal global, a diversidade de potência pode ser usada para fornecer multi-canais na recepção. As técnicas de equalização para os receptores PDR-MMSE e PDR-ZF são utilizadas para separar os termos dos símbolos transmitidos daqueles com interferência não-linear. $\mathrm{O}$ termo equalização aqui adotado refere-se ao cancelamento desta interferência.

Será visto que a utilização destas técnicas permite uma grande simplificação na estimação dos sinais de informação, com baixa complexidade computacional e fácil implementação. A estimação é realizada sobre o canal global sem que estejam disponíveis no receptor os parâmetros do PA. A principal vantagem do receptor PDR-MMSE é a sua maior robustez ao ruído em relação ao receptor PDR-ZF. Entretanto, ao utilizar o receptor PDR-MMSE, assume-se que a variância do ruído é conhecida, ao contrário do receptor PDR-ZF. Este, por sua vez, 


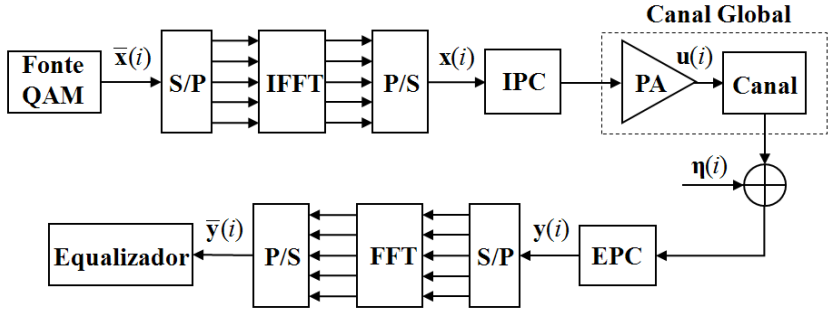

Fig. 1. Sistema OFDM discreto no tempo em banda base equivalente.

fornece uma estimação simplificada dos sinais de informação. Por outro lado, o método de equalização proposto apresenta uma taxa de transmissão dividida pelo fator de repetição dos símbolos. No entanto, tal fator pode ser reduzido em muitos casos, diminuindo seu efeito sobre a taxa de transmissão.

O artigo está estruturado de forma seguinte: na Seção II, o modelo para o sistema OFDM com amplificador não-linear é apresentado; na Seção III, a estimação do canal global nãolinear é introduzida; na Seção IV, um receptor baseado na diversidade de potência é desenvolvido; na Seção V uma análise dos resultados de simulação é fornecida e, finalmente, na Seção VI, as conclusões e perspectivas sobre o estudo são apresentadas.

\section{MODELO DE CANAL OFDM COM PA NÃO-LINEAR}

O sistema OFDM em banda base equivalente considerado neste trabalho é ilustrado através do diagrama de blocos da Fig. 1, em que uma seqüência de símbolos QAM (Quadrature Amplitude Modulation) independentes e identicamente distribuídos (i.i.d.) é gerada e enviada a um conversor serialparalelo (S/P) que os agrupa em blocos de $N$ símbolos cada, em que $N$ representa o número de sub-portadoras.

Neste trabalho considera-se que todas as variáveis com barra superior correspondem à representação de sinais no domínio da freqüência. Seja $\bar{x}_{n}(i)$, para $0 \leq n \leq N-1$, o símbolo QAM correspondente à $n$-ésima sub-portadora e ao $i$ ésimo bloco de transmissão, e $\overline{\mathbf{x}}(i)=\left[\begin{array}{lll}\bar{x}_{0}(i) & \ldots & \bar{x}_{N-1}(i)\end{array}\right]^{T}$ $\in \mathbb{C}^{N \times 1}$ o vetor contendo o $i$-ésimo bloco de $N$ símbolos QAM. É então realizada a IFFT (Inverse Fast Fourier Transform) sobre $\overline{\mathbf{x}}(i)$ :

$$
\mathbf{x}(i)=\mathbf{V}^{H} \overline{\mathbf{x}}(i)
$$

em que $\mathbf{x}(i)=\left[\begin{array}{lll}x_{0}(i) & \ldots & x_{N-1}(i)\end{array}\right]^{T} \in \mathbb{C}^{N \times 1}$ representa o $i$ ésimo bloco de sinais no domínio do tempo denominados símbolos OFDM, o sobrescrito $H$ representa a transposta do conjugado complexo (Hermitiana) e $\mathbf{V} \in \mathbb{C}^{N \times N}$ é a matriz de FFT (Fast Fourier Transform) dada por:

$$
\mathbf{V}=\frac{1}{\sqrt{N}}\left(\begin{array}{ccccc}
1 & 1 & 1 & \cdots & 1 \\
1 & w & w^{2} & \cdots & w^{(N-1)} \\
\vdots & \vdots & \vdots & \ddots & \vdots \\
1 & w^{(N-1)} & w^{2(N-1)} & \cdots & w^{(N-1)(N-1)}
\end{array}\right)
$$

em que $w=e^{-\frac{j 2 \pi}{N}}$.
Após a IFFT, um conversor paralelo - serial (P/S) organiza os blocos de $N$ símbolos OFDM em uma seqüência de símbolos OFDM para que seja introduzido um prefixo cíclico (IPC) de tamanho $M^{c p}$ entre os blocos. A inserção do prefixo cíclico evita a introdução de ISI (Inter Symbol Interference) entre os blocos de símbolos OFDM consecutivos, desde que $M^{c p} \geq M$, em que $M$ é a memória do canal sem fio. Embora implique em perda de potência e de banda, visto que mais dados serão transmitidos, esta solução computacionalmente eficiente resolve o problema da ISI entre os blocos de símbolo OFDM [7].

O símbolo OFDM é amplificado por um PA não-linear sem memória modelado por um polinômio de ordem $2 K-1$ mostrado a seguir [8]:

$$
u_{n}(i)=\sum_{k=0}^{K-1} a_{2 k+1}\left|x_{n}(i)\right|^{2 k} x_{n}(i),
$$

em que $u_{n}(i)$ representa o símbolo OFDM amplificado no $n$ ésimo instante $(0 \leq n \leq N-1)$ do $i$-ésimo bloco de transmissão, $a_{2 k+1}$, para $0 \leq k \leq K-1$, representam os coeficientes polinomiais do PA dados pelo vetor $\mathbf{a}=$ $\left[\begin{array}{ccc}a_{1} & \ldots & a_{2 K-1}\end{array}\right]^{T} \in \mathbb{C}^{K \times 1}$ e $K$ o número de coeficientes polinomiais. A Equação (3) pode ser reescrita como:

$$
u_{n}(i)=\sum_{k=0}^{K-1} a_{2 k+1} s_{2 k+1}\left\{x_{n}(i)\right\},
$$

em que $s_{2 k+1}\{\cdot\}$ é um operador definido como $s_{2 k+1}\left\{x_{n}(i)\right\}=$ $\left|x_{n}(i)\right|^{2 k} x_{n}(i)$. É observado não haver sinais associados aos termos pares do polinômio, pois são rejeitados pela filtragem em banda base equivalente.

Os sinais $u_{n}(i)$ em (4) podem ser representados também na forma vetorial como:

$$
\mathbf{u}(i)=\sum_{k=0}^{K-1} a_{2 k+1} \mathbf{s}_{2 k+1}\{\mathbf{x}(i)\},
$$

em que $\mathbf{u}(i)=\left[\begin{array}{lll}u_{0}(i) & \ldots & u_{N-1}(i)\end{array}\right]^{T} \in \mathbb{C}^{N \times 1}$ é o $i$-ésimo vetor de sinais na saída do PA e $\mathbf{s}_{2 k+1}\{\mathbf{x}(i)\}=\left[s_{2 k+1}\left\{x_{0}(i)\right\} \quad \ldots \quad s_{2 k+1}\left\{x_{N-1}(i)\right\}\right]^{T} \in \mathbb{C}^{N \times 1}$.

O sinal na saída do PA é então transmitido por um canal sem fio seletivo em frequiência e invariante no tempo de memória $M$ e resposta ao impulso $h_{m}$, para $0 \leq m \leq M$, representado pelo vetor $\mathbf{h}=\left[\begin{array}{llll}h_{0} & h_{1} & \ldots & h_{M}\end{array}\right]^{T} \in \mathbb{C}^{(M+1) \times 1}$. Seja o vetor de sinais recebidos após a extração do prefixo cíclico (EPC) dado por:

$$
\mathbf{y}(i)=\left[\begin{array}{lll}
y_{M^{c p}}(i) & \ldots & y_{N+M^{c p}}(i)
\end{array}\right]^{T} \in \mathbb{C}^{N \times 1},
$$

em que as componentes $y_{l}(i)$ para $0 \leq l \leq M^{c p}-1$ são descartadas. Desta forma, considerando um ruído branco gaussiano de média nula adicionado no receptor, o $i$-ésimo vetor de sinais recebido pode ser também expresso como:

$$
\mathbf{y}(i)=\mathbf{H u}(i)+\boldsymbol{\eta}(i)
$$


em que $\boldsymbol{\eta}(i)=\left[\begin{array}{lll}\eta_{0}(i) & \ldots & \eta_{N-1}(i)\end{array}\right]^{T} \in \mathbb{C}^{N \times 1}$ é o $i$-ésimo vetor de ruído e $\mathbf{H} \in \mathbb{C}^{N \times N}$ é uma matriz de convolução circular do canal sem fio [7].

Um conversor S/P agrupa novamente a seqüência de sinais no tempo por blocos de $N$ sinais para que seja realizada a FFT sobre (7), resultando em um vetor de sinais no domínio da freqüência $\overline{\mathbf{y}}(i)=\left[\bar{y}_{0}(i) \quad \ldots \quad \bar{y}_{N-1}(i)\right]^{T} \in \mathbb{C}^{N \times 1}$ dado por:

$$
\overline{\mathbf{y}}(i)=\boldsymbol{\Lambda} \overline{\mathbf{u}}(i)+\overline{\mathbf{\eta}}(i) \text {, }
$$

em que $\overline{\boldsymbol{\eta}}(i)=\left[\begin{array}{lll}\bar{\eta}_{0}(i) & \ldots & \bar{\eta}_{N-1}(i)\end{array}\right]^{T} \in \mathbb{C}^{N \times 1}$ é o vetor de ruído no domínio da freqüência, $\boldsymbol{\Lambda}=\mathbf{V H V}^{H}=\operatorname{diag}[\lambda] \epsilon$ $\mathbb{C}^{N \times N}$ é uma matriz diagonal [15] contendo as amostras da resposta em frequiência do canal sem fio representadas pelo vetor $\lambda=\left[\begin{array}{lll}\lambda_{0} & \ldots & \lambda_{N-1}\end{array}\right]^{T} \in \mathbb{C}^{N \times 1}$ e $\overline{\mathbf{u}}(i)=\left[\begin{array}{ll}\bar{u}_{0}(i) & \ldots\end{array}\right.$ $\left.\bar{u}_{N-1}(i)\right]^{T} \in \mathbb{C}^{N \times 1} \in \mathbb{C}^{N \times 1}$ é a versão em freqüência do vetor de sinais na saída do PA, em que, de (2) e (5), é escrito por:

$$
\overline{\mathbf{u}}(i)=\sum_{k=0}^{K-1} a_{2 k+1} \overline{\mathbf{s}}_{2 k+1}\{\mathbf{x}(i)\}
$$

sendo $\overline{\mathbf{s}}_{2 k+1}\{\mathbf{x}(i)\}=\mathbf{V s}_{2 k+1}\{\mathbf{x}(i)\} \in \mathbb{C}^{N \times 1}$.

Substituindo (9) em (8), o vetor de sinais recebidos $\overline{\mathbf{y}}(i)$ no domínio da frequiência tem como componentes os sinais recebidos $\bar{y}_{n}(i)$ na $n$-ésima sub-portadora no $i$-ésimo bloco de transmissão dado por:

$$
\bar{y}_{n}(i)=\sum_{k=0}^{K-1} \lambda_{n} a_{2 k+1} \bar{s}_{n, 2 k+1}\{\mathbf{x}(i)\}+\bar{\eta}_{n}(i),
$$

em que $\overline{\mathrm{s}}_{\mathrm{n}, 2 \mathrm{k}+1}\{\mathrm{x}(\mathrm{i})\}$ representa o n-ésimo elemento do vetor $\overline{\mathrm{s}}_{2 \mathrm{k}+1}\{\mathrm{x}(\mathrm{i})\}$. Definindo um canal global que compreende conjuntamente os coeficientes do canal sem fio e do PA contidos em (10), na forma:

$$
g_{n, 2 k+1}=\lambda_{n} a_{2 k+1},
$$

para $0 \leq n \leq N-1$ e $0 \leq k \leq K-1$. Substituindo (11) em (10), resulta em (12), mostrada a seguir, na qual cada termo contém um único fator que representa de forma sintética a influência conjunta do canal sem fio na $n$-ésima sub-portadora e do amplificador:

$$
\bar{y}_{n}(i)=\sum_{k=0}^{K-1} g_{n, 2 k+1} \bar{s}_{n, 2 k+1}\{\mathbf{x}(i)\}+\bar{\eta}_{n}(i) .
$$

A Equação (12) mostra que o sinal recebido no domínio da freqüência $\bar{y}_{n}(i)$ é composto por uma versão amplificada e rotacionada de $\bar{x}_{n}(i)$, além das interferências ICI e ruído. Observa-se que o termo $\bar{s}_{n, 2 k+1}\{\mathbf{x}(i)\}$ depende dos símbolos de informação em outras sub-portadoras.

Note também que $\bar{y}_{n}(i)$ não é contaminado com interferências de outros símbolos de informação $\bar{x}_{n^{\prime}}(i)$, para $n^{\prime} \neq n$. Assim, o cancelamento da ICI deve ser feito por um equalizador no domínio da freqüência, que deve eliminar os termos de ICI presentes em $\sum_{k=1}^{K-1} g_{n, 2 k+1} \bar{s}_{n, 2 k+1}\{\mathbf{x}(i)\}$, além de remover o fator escalar $g_{n, 1}$.
Seja um conjunto de $I_{B}$ blocos de transmissão e $\overline{\mathbf{y}}_{n}=$ $\left[\bar{y}_{n}(0) \quad \ldots \quad \bar{y}_{n}\left(I_{B}-1\right)\right] \in \mathbb{C}^{1 \times I_{B}}$ o vetor contendo os $I_{B}$ sinais recebidos ao longo do tempo no domínio da freqüência na $n$-ésima sub-portadora. A Equação (12), sem ruído, pode então ser escrita na forma vetorial como:

$$
\overline{\mathbf{y}}_{n}=\mathbf{g}_{n}^{T} \overline{\mathbf{S}}_{n}
$$

em que $\mathbf{g}_{n}=\left[\begin{array}{lll}g_{n, 1} & \cdots & g_{n, 2 k+1}\end{array}\right]^{T} \in \mathbb{C}^{K \times 1}$ é o vetor contendo os coeficientes do canal global na $n$-ésima sub-portadora e $\overline{\mathbf{S}}_{n} \in \mathbb{C}^{K \times I_{B}}$ representa uma matriz dada por:

$$
\overline{\mathbf{S}}_{n}=\left(\begin{array}{ccc}
\bar{s}_{n, 1}\{\mathbf{x}(0)\} & \cdots & \bar{s}_{n, 1}\left\{\mathbf{x}\left(I_{B}-1\right)\right\} \\
\vdots & \ddots & \vdots \\
\bar{s}_{n, 2 K-1}\{\mathbf{x}(0)\} & \cdots & \bar{s}_{n, 2 K-1}\left\{\mathbf{x}\left(I_{B}-1\right)\right\}
\end{array}\right) .
$$

As equações (12) e (13) mostram a dependência linear entre o sinal recebido e os coeficientes do canal global, que podem ser estimados por meio de um estimador de Mínimos Quadrados (LS) utilizando-se $I_{P}$ símbolos QAM pilotos (conhecidos) por sub-portadora durante um período de treinamento, como será visto na Seção III. Após a estimação do canal global, um equalizador no domínio da freqüência é proposto para cancelar a ICI introduzida pelo PA no sinal transmitido.

\section{ESTIMAÇÃO DE CANAL}

Deseja-se estimar os coeficientes do canal global (13) que compreende os coeficientes do canal sem fio e do PA. A estimação empregada é baseada em um método do tipo LS que utiliza blocos com $I_{P}$ símbolos pilotos por sub-portadora durante um período de treinamento dados pelo vetor $\overline{\mathbf{x}}_{n}^{(P)}=$ $\left[\bar{x}_{n}^{(P)}(0) \quad \ldots \quad \bar{x}_{n}^{(P)}\left(I_{P}-1\right)\right] \in \mathbb{C}^{1 \times I_{P}}$, para $0 \leq i \leq I_{P}-1 \mathrm{e}$ $0 \leq n \leq N-1$ em que se considera $I_{P} \geq K$ e assume-se neste item que os símbolos pilotos são alocados em todas as subportadoras, por razões de simplicidade e precisão. É importante ressaltar que se pode também utilizar uma estratégia em que os símbolos pilotos são alocados apenas em algumas sub-portadoras. Assim, a estimação dos coeficientes do canal global nas sub-portadoras sem símbolos pilotos é feita através de técnicas de interpolação [16], o que resulta em uma economia no tempo de transmissão dos símbolos pilotos. Neste trabalho, os efeitos da interpolação, a qual utiliza a FFT [16], são avaliados no item VI através de resultados obtidos por simulações.

Seja $\overline{\mathbf{y}}_{n}^{(P)}=\left[\bar{y}_{n}^{(P)}(0) \quad \ldots \quad \bar{y}_{n}^{(P)}\left(I_{P}-1\right)\right] \in \mathbb{C}^{1 \times I_{P}}$ o vetor de sinais recebidos no domínio da frequêencia com $I_{P}$ símbolos pilotos na $n$-ésima sub-portadora. Utilizando-se (13), este vetor pode ser expresso da seguinte forma:

$$
\overline{\mathbf{y}}_{n}^{(P)}=\mathbf{g}_{n}^{T} \overline{\mathbf{S}}_{n}^{(P)}
$$

em que $\overline{\mathbf{S}}_{n}^{(P)} \in \mathbb{C}^{K \times I_{P}}$ é uma matriz dada a seguir, a partir de (14) :

$$
\overline{\mathbf{S}}_{n}^{(P)}=\left(\begin{array}{ccc}
\bar{s}_{n, 1}\left\{\mathbf{x}^{(P)}(0)\right\} & \cdots & \bar{s}_{n, 1}\left\{\mathbf{x}^{(P)}\left(I_{P}-1\right)\right\} \\
\vdots & \ddots & \vdots \\
\bar{s}_{n, 2 K-1}\left\{\mathbf{x}^{(P)}(0)\right\} & \cdots & \bar{s}_{n, 2 K-1}\left\{\mathbf{x}^{(P)}\left(I_{P}-1\right)\right\}
\end{array}\right),
$$


em que $x_{n}^{(P)}(i)$ é a versão no domínio do tempo de $\bar{x}_{n}^{(P)}(i)$, para $0 \leq n \leq N-1$.

A estimação LS dos coeficientes do canal global $\mathbf{g}_{n}$ pode ser dada por:

$$
\hat{\mathbf{g}}_{n}=\left[\overline{\mathbf{y}}_{n}^{(P)}\left[\overline{\mathbf{S}}_{n}^{(P)}\right]^{\dagger}\right]^{T},
$$

em que $\overline{\mathbf{y}}_{n}^{(P)}$ e $\overline{\mathbf{S}}_{n}^{(P)}$ são dados por (15) e (16), respectivamente, para $0 \leq n \leq N-1$, e $(\cdot)^{\dagger}$ denota a matriz pseudo-inversa. Destaca-se que o canal global estimado em (17) supõe a sua invariância temporal durante $I_{P}$ blocos formados por símbolos pilotos. De fato, este canal estimado é também suposto invariante no tempo para os $I_{B}$ blocos seguintes, no que concerne à equalização realizada neste período. Portanto, a estratégia de estimação aqui adotada supõe a invariância temporal do canal global durante a transmissão de cada grupo de $\left(I_{P}+I_{B}\right)$ blocos de dados.

É importante destacar que o ruído não foi considerado para efeito da escolha do melhor estimador de canal global. No entanto, espera-se que o método empregado diminua essa importância caso seja possível aumentar o número de blocos usados em cada estimação.

\section{RECEPTOR BASEADO NA DIVERSIDADE DE POTÊNCIA}

Propõem-se nesta sessão duas técnicas para a recuperação da informação, ambas considerando a re-transmissão dos símbolos de informação $\bar{x}_{n}(i)$ com potências diferentes, assumindo que os coeficientes $g_{n, 2 k+1}$ são conhecidos de forma precisa ou estimados à partir de (17).

\section{A. Modelo do sinal em freqüência para a transmissão com repetição}

O símbolo transmitido no domínio da freqüência na $n$ ésima sub-portadora, para $0 \leq i \leq I_{B}-1$ e $0 \leq l \leq L-1$, é dado por

$$
\bar{x}_{n}^{(p d)}(i L+l)=\sqrt{P_{l}} \bar{x}_{n}(i),
$$

em que $\bar{x}_{n}^{(p d)}(p)$ é o $p$-ésimo símbolo transmitido no domínio da freqüência, para $p=i L+l$ e $0 \leq p \leq I_{B} L-1, L$ é o fator de repetição e $P_{l}$ é a potência de transmissão.

Note que, para cada sub-portadora, um símbolo de informação no domínio da freqüência $\bar{x}_{n}(i)$ gera um grupo de $L$ símbolos transmitidos no domínio do freqüência $\bar{x}_{n}^{(p d)}(i L+$ l) e, da mesma forma, um grupo de $L$ sinais recebidos no domínio do freqüência $\bar{y}_{n}^{(p d)}(i L+l)$. Seja $\overline{\mathbf{y}}_{n}^{(p d)}(i)=$ $\left[\bar{y}_{n}^{(p d)}(i L+0) \quad \ldots \quad \bar{y}_{n}^{(p d)}((i+1) L-1)\right]^{T} \in \mathbb{C}^{L \times 1} \quad$ o vetor contendo os $L$ sinais recebidos no domínio da freqüência na $n$ ésima sub-portadora associados ao $i$-ésimo símbolo de informação no domínio da freqüência $\bar{x}_{n}(i)$. A Equação (13) é usada, considerando a sequiência dos símbolos repetidos, resultando na forma:

$$
\overline{\mathbf{y}}_{n}^{(p d)}(i)=\left[\overline{\mathbf{S}}_{n}^{(p d)}(i)\right]^{T} \mathbf{g}_{n}
$$

em que a matriz $\overline{\mathbf{S}}_{n}^{(p d)}(i) \in \mathbb{C}^{K \times L}$ é definida, se inspirando em (14) ou (16), como:

$$
\overline{\mathbf{S}}_{n}^{(p d)}(i)=\left(\begin{array}{ccc}
\bar{s}_{n, 1}\left\{\mathbf{x}^{(p d)}(i L)\right\} & \cdots & \bar{s}_{n, 1}\left\{\mathbf{x}^{(p d)}((i+1) L-1)\right\} \\
\vdots & \ddots & \vdots \\
\bar{s}_{n, 2 K-1}\left\{\mathbf{x}^{(p d)}(i L)\right\} & \cdots & \bar{s}_{n, 2 K-1}\left\{\mathbf{x}^{(p d)}((i+1) L-1)\right\}
\end{array}\right),
$$

Utilizando (18), a matriz $\overline{\mathbf{S}}_{n}^{(p d)}(i)$ em (20) pode ser reescrita como

$$
\overline{\mathbf{S}}_{n}^{(p d)}(i)=\operatorname{diag}\left[\overline{\mathbf{s}}_{n}^{(p d)}(i)\right] \mathbf{P}^{T},
$$

em que $\overline{\mathbf{s}}_{n}^{(p d)}(i)=\left[\bar{s}_{1}\left\{x_{n}(i)\right\} \quad \ldots \quad \bar{s}_{2 K-1}\left\{x_{n}(i)\right\}\right]^{T} \in \mathbb{C}^{K \times 1} \mathrm{e}$

$$
\mathbf{P}=\left(\begin{array}{ccc}
P_{0}^{\frac{1}{2}} & \cdots & P_{0}^{\frac{2 K-1}{2}} \\
\vdots & \ddots & \vdots \\
P_{L-1}^{\frac{1}{2}} & \cdots & P_{L-1}^{\frac{2 K-1}{2}}
\end{array}\right) \in \mathbb{C}^{L \times K}
$$

Substituindo (21) em (19), obtém-se

$$
\overline{\mathbf{y}}_{n}^{(p d)}(i)=\operatorname{Pdiag}\left[\mathbf{g}_{n}\right] \overline{\mathbf{s}}_{n}^{(p d)}(i)
$$

Definindo-se a matriz $\overline{\mathbf{Y}}_{n}^{(p d)} \in \mathbb{C}^{L \times I_{B}}$, cujas colunas são os vetores no domínio da freqüência $\overline{\mathbf{y}}_{n}^{(p d)}(i)$, para $0 \leq n \leq N-1$, e usando (22), escreve-se a matriz de vetores dos sinais recebidos no domínio da frequiência em $I_{B}$ instantes:

$$
\overline{\mathbf{Y}}_{n}^{(p d)}=\mathbf{P} \operatorname{diag}\left[\mathbf{g}_{n}\right] \overline{\mathbf{S}}_{n},
$$

em que $\overline{\mathbf{S}}_{n} \in \mathbb{C}^{K \times I_{B}}$ é definido em (14) e $0 \leq n \leq N-1$.

A análise de (23) com (13), indica que, ao re-transmitir os símbolos $\bar{x}_{n}(i) L$ vezes, com diferentes amplitudes, cria-se o efeito de $L$ sub-canais para o sistema OFDM não-linear, cuja matriz de canal equivalente é dada por (Pdiag $\left.\left[\mathbf{g}_{n}\right]\right)$. Pode-se então concluir que a variação de potência por repetição de símbolos seja vista como uma diversidade para os sinais recebidos.

\section{B. Equalização em freqüência de canal não-linear}

Dada a representação de multicanal descrita em (23), os sinais de informação no domínio da frequiência $\bar{x}_{n}(i)$ podem ser extraídos da matriz $\overline{\mathbf{Y}}_{n}^{(p d)}$ de sinais recebidos no domínio da freqüência por meio de técnicas de processamento multicanal. Diversas técnicas podem ser usadas nos receptores para este propósito, tais como aquelas que fazem uso de equalizadores no domínio da freqüência com critérios MMSE e ZF. Serão denominados de receptores PDR-MMSE e PDR$\mathrm{ZF}$ às estratégias que fazem uso daqueles equalizadores, respectivamente, nos sistemas OFDM com repetição de símbolos com potências diferentes. Neste caso, após algumas operações, a partir de (23), os coeficientes dos equalizadores nos receptores PDR-MMSE e PDR-ZF são dados respectivamente por

$$
\begin{gathered}
\mathbf{W}_{n}=\mathbf{R}_{\overline{\mathbf{S}}} \operatorname{diag}\left[\mathbf{g}_{n}^{*}\right] \mathbf{P}^{H}\left(\mathbf{P} \operatorname{diag}\left[\mathbf{g}_{n}\right] \mathbf{R}_{\overline{\mathbf{S}}} \operatorname{diag}\left[\mathbf{g}_{n}^{*}\right] \mathbf{P}^{H}+\mathbf{I}_{L} \sigma^{2}\right)^{-1}, \\
\mathbf{W}_{n}=\left(\mathbf{P} \operatorname{diag}\left[\mathbf{g}_{n}\right]\right)^{\dagger},
\end{gathered}
$$

em que $\mathbf{W}_{n} \in \mathbb{C}^{K \times L}, \sigma^{2}$ é a variância do ruído e $\mathbf{R}_{\overline{\mathbf{s}}}=$ $\mathbb{E}\left[\overline{\mathbf{x}}_{n}(i) \overline{\mathbf{x}}_{n}^{H}(i)\right] \in \mathbb{C}^{K \times K}$ é a matriz de covariância do vetor 
$\overline{\mathbf{s}}_{n}^{(p d)}(i)$ e $0 \leq n \leq N-1$. É observado que, a expressão (24) se reduz à (25) quando $\sigma^{2}=0$, como esperado.

A matriz $\mathbf{W}_{n}$ representa a operação de equalização no domínio da freqüência sobre $\overline{\mathbf{Y}}_{n}^{(p d)}$ no receptor, a qual reduz a ICI e se assemelha à operação de equalização no domínio do tempo por filtragem em receptores de canais seletivos em freqüência para a redução ou cancelamento de ISI.

Desta forma, a matriz $\overline{\mathbf{S}}_{n}$ de sinais estimados na freqüência no período $I_{B}$ é dada por:

$$
\hat{\mathbf{S}}_{n}=\mathbf{W}_{n} \overline{\mathbf{Y}}_{n}^{(p d)}
$$

na qual $0 \leq n \leq N-1$. Os sinais estimados em (26) são submetidos a dispositivos de decisão por limiar para cada frequiência, os quais fornecem a seqüência de símbolos estimados no período $I_{B}$. Como se observa de (14) e (16), a primeira linha de $\hat{\overline{\mathbf{S}}}_{n}$ corresponde aos termos lineares, representando os símbolos $\tilde{\bar{x}}_{n}(i)$ estimados no domínio da freqüência. As demais linhas representam a estimação de termos não-lineares dos símbolos estimados, os quais podem ser descartados.

A principal vantagem de um receptor PDR-MMSE é que ele é mais robusto ao ruído do que o receptor PDR-ZF. Entretanto, ao utilizar o receptor PDR-MMSE, assume-se que a variância do ruído seja conhecida, ao contrario do receptor PDR-ZF. Além disso, como é mostrado a seguir, o receptor PDR-ZF permite uma simplificação computacional importante na estimação dos sinais de informação $\bar{x}_{n}(i)$. No receptor PDR-ZF, ao substituir-se (25) em (26), obtém-se:

$$
\hat{\overline{\mathbf{S}}}_{n}=\operatorname{diag}\left[\zeta\left(\mathbf{g}_{n}\right)\right] \mathbf{P}^{\dagger} \overline{\mathbf{Y}}_{n}^{(p d)} \in \mathbb{C}^{K \times I_{B},}
$$

em que $\zeta\left(\mathbf{g}_{n}\right)$ é uma operação que inverte (ponto-a-ponto) cada elemento do vetor $\mathbf{g}_{n}$ no argumento. De fato, denotado por $\widetilde{\mathbf{p}} \in \mathbb{C}^{1 \times L}$ a primeira linha de $\mathbf{P}^{\dagger}$, a primeira linha de $\hat{\overline{\mathbf{S}}}_{n}$ em (27), denotada por $\left[\hat{\mathbf{S}}_{n}\right]_{1, \cdot}$ pode ser expressa como

$$
\left[\hat{\overline{\mathbf{S}}}_{n}\right]_{1, \cdot}=\frac{\widetilde{\mathbf{p}}}{g_{n, 1}} \overline{\mathbf{Y}}_{n}^{(p d)} \in \mathbb{C}^{1 \times I_{B}} .
$$

A partir de (28), a estimação dos sinais de informação nas $N$ sub-portadoras e nos $I_{B}$ blocos de transmissão pode ser escrita em uma forma compacta dada por:

$$
\hat{\overline{\mathbf{s}}}=\widetilde{\mathbf{p}} \overline{\mathbf{Y}}^{(p d)} \operatorname{diag}\left(\zeta\left(\mathbf{g}^{(l i n)}\right) \otimes \mathbf{1}_{I_{B}}\right),
$$

em que $\otimes$ denota o produto de Kronecker, $\mathbf{1}_{I_{B}} \in \mathbb{R}^{I_{B} \times 1}$ é um vetor de dimensão $I_{B}$ em que todos os elementos são 1 , $\mathbf{g}^{(l i n)}=\left[\begin{array}{lll}g_{0,1} & \ldots & g_{N-1,1}\end{array}\right]^{T} \in \mathbb{C}^{N \times 1}, \overline{\mathbf{Y}}^{(p d)}=\left[\begin{array}{lll}\overline{\mathbf{Y}}_{0}^{(p d)} & \ldots & \overline{\mathbf{Y}}_{N-1}^{(p d)}\end{array}\right] \in$ $\mathbb{C}^{L \times I_{B} N}$ e $\hat{\overline{\mathbf{s}}}=\left[\left[\hat{\overline{\mathbf{S}}}_{0}\right]_{1,} \quad \cdots \quad\left[\hat{\overline{\mathbf{S}}}_{N-1}\right]_{1,}\right] \in \mathbb{C}^{1 \times I_{B} N}$.

Por outro lado, os $I_{B}$ símbolos estimados por sub-portadora no receptor PDR-MMSE são obtidos da primeira linha de $\hat{\overline{\mathbf{S}}}_{n}$, substituindo (24) em (26), fornecendo:

$\hat{\overline{\mathbf{S}}}_{n}=\mathbf{R}_{\overline{\mathbf{S}}} \operatorname{diag}\left[\mathbf{g}_{n}^{*}\right] \mathbf{P}^{H}\left(\mathbf{P} \operatorname{diag}\left[\mathbf{g}_{n}\right] \mathbf{R}_{\overline{\mathbf{S}}} \operatorname{diag}\left[\mathbf{g}_{n}^{*}\right] \mathbf{P}^{H}+\mathbf{I}_{L} \sigma^{2}\right)^{-1} \overline{\mathbf{Y}}_{n}^{(p d)}(30)$

Note que, a condição necessária para implementação das técnicas propostas é que $\mathbf{P}$ tenha posto-coluna completo, que significa que o fator de repetição $L$ deve satisfazer $L \geq K$. Esta técnica diminui a taxa de transmissão em $L$ vezes. Entretanto, assumindo $K=2$ (polinômio não-linear de terceira ordem), pode-se usar $L=2$. Assim, os resultados de simulação, mostram que esta limitação da técnica é compensada por seu bom desempenho.

Uma característica importante ainda a destacar no receptor PDR-ZF é que são utilizados somente os coeficientes $g_{n, 1}$ associados aos termos lineares. Isto significa que não é preciso conhecer os coeficientes do PA, com exceção do coeficiente linear $a_{1}$. Além disso, quando $K=L=2$, a técnica ZF necessita somente do cálculo de uma única matriz inversa de dimensões $2 \times 2$, válida para todas as sub-portadoras $(0 \leq$ $n \leq N-1$ e $\left.0 \leq i \leq I_{B}-1\right)$, diferente do receptor PDRMMSE, o qual calcula uma matriz inversa para cada subportadora, conforme indicado em (30).

\section{RESUltAdOS DE SIMULAÇÃO}

Nesta seção avalia-se o desempenho das técnicas adotadas através de simulações computacionais que utilizam 100 realizações independentes de Monte Carlo. O sistema OFDM com prefixo cíclico de comprimento 4 possui $N=64$ subportadoras e um PA não-linear de terceira ordem com coeficientes $a_{1}=0,9798-0,2887 j$ e $a_{3}=-0,2901+$ $0,4350 j$, em que $I_{B}=50$ blocos de transmissão contendo símbolos 64-QAM são transmitidos por um canal sem fio multi-percurso seletivo em freqüência de memória $M=4$, cujos coeficientes têm distribuição Rayleigh mutuamente independentes, com média zero, variância unitária, e são mantidos constantes a cada realização. Para a estimação do canal global, utilizaram-se $I_{P}=2$ símbolos pilotos em cada uma de 16 sub-portadoras regularmente espaçadas na banda do canal. Ademais, para ambos receptores PDR- (ZF, MMSE), foi utilizado um fator de repetição $L=2$ com potências de transmissão $P_{0}=1$ e $P_{1}=0,64$. Os resultados obtidos são analisados por meio da taxa de erro de bits (TEB) em função da SNR (Signal-to-Noise Ratio).

A Fig. 2 mostra a TEB associada aos símbolos decididos nos receptores PDR-ZF e PDR-MMSE, em que se considera os coeficientes dos canais globais estimados (CGE). Nesta figura é também mostrada a influência do PA sem equalização e com amplificação: linear $\left(a_{3}=0\right)$ e não-linear (NL). Neste caso, os sinais recebidos foram multiplicados pelo fator de ganho complexo $1 / g_{n, 1}$, considerando-se o canal global conhecido (CGC).

Observa-se um desempenho inaceitável para o sistema OFDM sem uma contramedida para se opor às distorções provocadas pela não-linearidade do amplificador quando comparado ao sistema com amplificação somente linear. A presença de ambos equalizadores reduz a ICI melhorando o desempenho do sistema. O receptor PDR-MMSE, para uma baixa SNR, possui um melhor desempenho que o receptor PDR-ZF, pois é mais robusto ao ruído, sendo ainda comparável ao OFDM linear. No caso do ruído desprezível, os 
dois receptores possuem o mesmo desempenho, conforme esperado. No entanto, para alta SNR, ambos receptores têm desempenho inferior ao OFDM com PA linear, e superior ao PA não-linear. Os desempenhos se assemelham para baixa SNR.

Na Fig. 3, o desempenho dos receptores PDR-ZF e PDRMMSE é comparado considerando os sinais equalizados para CGE e CGC. São mostradas as curvas da TEB associada aos símbolos decididos, fazendo uso de CGC e CGE. Observa-se, em geral, uma boa aproximação entre as curvas dos dois receptores, respectivamente, para CGC e CGE, indicando que a técnica de estimação proposta gera bons resultados. No entanto, verificou-se da figura que a saturação na TEB para alta SNR é provocada pelos erros de estimação de canal global. Novamente, quando o ruído é desprezível, os dois receptores possuem o mesmo desempenho.

\section{CONCLUSÃO E PERSPECTIVAS}

Este trabalho apresentou técnicas de estimação e equalização em freqüência de canal não-linear para reduzir ou cancelar a ICI dos sinais recebidos em sistemas OFDM oriunda de amplificadores no transmissor. As técnicas utilizadas são baseadas na representação de um canal global que compreende a cascata de um amplificador no transmissor com um canal linear sem fio seletivo em frequiência. A técnica de estimação do canal emprega uma aproximação LS do canal global. As técnicas de equalização propostas utilizam processamento de sinais por blocos nos receptores PDRMMSE e PDR-ZF. A diversidade de potência, adotada no sistema através da re-transmissão dos símbolos com potências diferentes, combate eficazmente à ICI sob o custo da diminuição de capacidade do sistema OFDM por subportadora. Nos próximos trabalhos, as técnicas aqui propostas serão aplicadas para sistemas MIMO, bem como serão realizadas comparações com outras técnicas de compensação de não-linearidades em sistemas OFDM, e serão desenvolvidos novos estimadores para evitar a saturação da TEB para SNR alta e novas estratégias para aumento da capacidade, além de considerar não-linearidades de maior ordem.

\section{REFERÊNCIAS BIBLIOGRÁFICAS}

[1] P. Banelli, G. Baruffa, and S. Cacopardi. "Effects of HPA nonlinearity on frequency multiplexed OFDM signals". IEEE Transactions on Broadcasting, 47(2):123-136, Jun. 2001

[2] P. Banelli and S. Cacopardi. "Theoretical analysis and performance of OFDM signals in nonlinear AWGN channels". IEEE Transactions on Communications, 48(3):430-441, Mar. 2000.

[3] E. Costa and S. Pupolin. "M-QAM-OFDM system performance in the presence of a nonlinear amplifier and phase noise". IEEE Transactions on Communications, 50(3):462-472, Mar. 2002.

[4] D. Dardari, V. Tralli, and A. Vaccari. "A theoretical characterization of nonlinear distortion effects in OFDM systems". IEEE Transactions on Communications, 48(10):1755-1764, Oct. 2000.

[5] A. N. D'Andrea, V. Lottici, and R. Reggiannini. "Nonlinear predistortion of OFDM signals over frequency-selective fading channels". IEEE Transactions on Communications, 49(5):837-843, May 2001.

[6] A. J. Redfern and G. T. Zhou. "Nonlinear channel identification and equalization for OFDM systems". In IEEE International Conference on Acoustics, Speech and Signal Processing, volume 6, pages 3521-3524, Seattle, WA,USA, May 1998.
[7] P. P. Vaidyanathan and B. Vrcelj, "Fast and robust blind-equalization based on cyclic prefix," in Proc. IEEE Int. Conf. on Comm., pages 1-5, New York, AprMay 2002.

[8] L. Ding. "Digital Predistortion of Power Amplifiers for Wireless Applications". $\mathrm{PhD}$ thesis, School of Electrical and Computer Engineering, Georgia Institute of Technology, USA, Mar. 2004.

[9] A. N. D'Andrea, V. Lottici, and R. Reggiannini. "Nonlinear predistortion of OFDM signals over frequency-selective fading channels". IEEE Transactions on Communications, 49(5):837-843, May 2001.

[10] L. Ding, G. T. Zhou, D. R. Morgan, Z. Ma, J. S. Kenney, J. Kim, and C. R. Giardina. "A robust digital baseband predistorter constructed using memory polynomials". IEEE Transactions on Communications, 52(1):159-165, Jan. 2004.

[11] R. Lopez-Valcarce and S. Dasgupta. "Blind equalization of nonlinear channels from second-order statistics". IEEE Transactions on Signal Processing, 49(12):3084-3097, Dec. 2001

[12] N.Y. Ermolova. "OFDM equalization in nonlinear time-varying channels". In International Symposium on Wireless Communication Systems, pages 358-362, Valencia Spain, Sep. 2006.

[13] N.Y. Ermolova, N. Nefedov, and S. Haggman. "An iterative method for nonlinear channel equalization in OFDM systems". In IEEE International Symposium on Personal, Indoor and Mobile Radio Communications, volume 1, pages 484-488, Barcelona, Spain, Sep. 2004.

[14] S. Litsyn. "Peak Power Control in Multicarrier Communications". Cambridge University Press, 1st edition, 2007.

[15] R. M. Gray. "Toeplitz and circulant matrices: A review. Foundations and Trends in Communications and Information Theory", 2(3):155-239, 2005.

[16] F. Gregorio, S. Werner, T. I. Laakso, and J. Cousseau. "Receiver cancellation technique for nonlinear power amplifier distortion in SDMA-OFDM systems". IEEE Transactions on Vehicular Technology, 56(5):2499-2516, Sep. 2007.

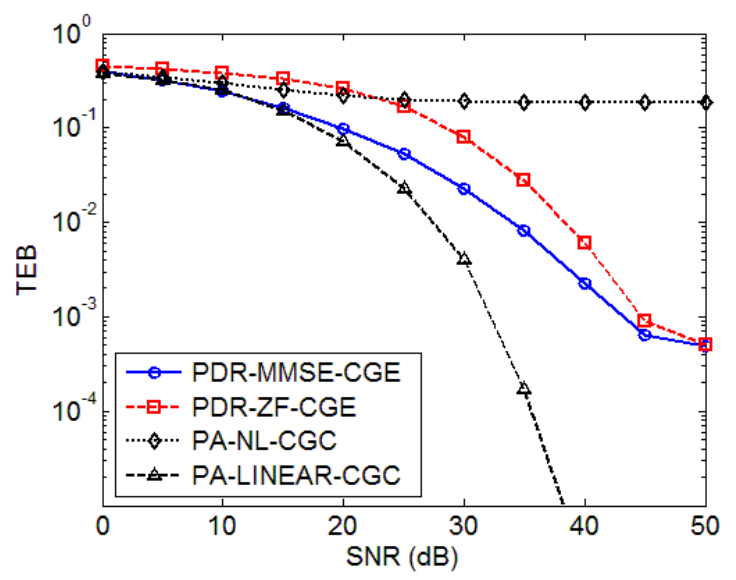

Fig. 2. TEB do sistema OFDM com e sem equalização.

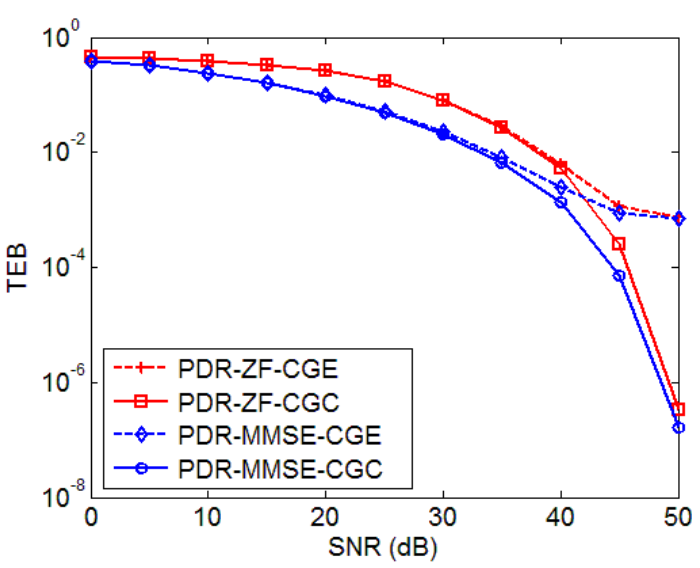

Fig. 3. TEB do sistema OFDM equalizado com canal global estimado (PDRZF, MMSE-CGE) e com canal global conhecido (PDR-ZF, MMSE-CGC). 